REVIEW ARTICLE

\title{
Non-Cannabinoid Metabolites of Cannabis sativa with Therapeutic Potential
}

\author{
Henry Lowe ${ }^{1,2,3,4}$, Blair Steele ${ }^{1^{*}}$, Joseph Bryant ${ }^{1}$, Ngeh Toyang $^{2,3}$ and Wilfred F. Ngwa ${ }^{5}$ \\ $1 \quad$ Biotech R \& D Institute, University of the West Indies, Mona, Jamaica; \\ blairgsteele@gmail.com (B.S.) \\ 2 Vilotos Pharmaceuticals Inc., Baltimore, MD, 21202, United States; \\ lowebiotech@gmail.com (H.L.); ngeh.toyang@flavocure.com (N.T.). \\ $3 \quad$ Flavocure Biotech Inc., Baltimore, MD, 21202, United States. \\ $4 \quad$ University of Maryland Medical School, Baltimore, MD, 21202, United States. \\ 5 Brigham and Women's Hospital, Dana-Faber Cancer Institute, Harvard Medical \\ School, Boston, MA, United States. \\ *Correspondence blairgsteele@gmail.com; Tel.: 876-926-8502
}

\begin{abstract}
The Cannabis plant (Cannabis sativa L.) produces an estimated 545 chemical compounds of different biogenetic classes. In addition to economic value, many of these phytochemicals have medicinal and physiological activity. The plant is most popularly known for its two most prominent and most studied secondary metabolites-

$\Delta^{9}$-Tetrahydrocannabinol ( $\Delta^{9}$-THC) and Cannabidiol (CBD). Both $\Delta^{9}$-THC and CBD have a wide therapeutic window across many ailments and form part of a class of secondary metabolites called cannabinoids - of which approximately over 104 exist. This review will focus on noncannabinoid metabolites of Cannabis sativa that also have therapeutic potential, some of which share medicinal properties similar to those of cannabinoids. The most notable of these noncannabinoid phytochemicals are flavonoids and terpenes. We will also discuss future directions in cannabis research and development of cannabis-based pharmaceuticals. Caflanone, a flavonoid molecule with selective activity against the human viruses including the coronavirus SARS-COV2, and certain cancers, is one of the most promising non-cannabinoid molecules that is being advanced into clinical trials. As validated by thousands of years of the use of cannabis for medicinal purposes, vast anecdotal evidence abounds on the medicinal benefits of the plant. These benefits are attributed to the many phytochemicals in this plant, including noncannabinoids. The most promising non-cannabinoids with potential to alleviate global disease burdens are discussed.
\end{abstract}

Keywords: cannabinoids; $\Delta^{9}$-tetrahydrocannabinol, cannabidiol, non-cannabinoids, flavonoids; terpenes; secondary metabolites; 


\section{Introduction}

A central Asian site of domestication of the cannabis plant is often cited with the plant's medicinal value first recorded in the Pen Ts'ao Ching, the Chinese pharmacopoeia-the world's oldest, compiled in the Han dynasty, first or second century A.D. [1]. The cannabis plant, its resin, and some derivatives of its compounds have been used in traditional eastern medicine. Uses include: for recreation due to its hallucinogenic/hypnotic effects, for religious celebrations and meditative purposes; industrially for fibers, textiles and ropes, and medicinally as an analgesic anti-convulsant, antidiarrheal, sedative, relaxant, anxiolytic, antibacterial, antioxidant, and as treatment for tetanus, epilepsy and delirium tremens.

The Cannabis plant produces an estimated 545 [2] chemical compounds of different biogenetic classes [3]. These include primary metabolites, like amino acids, fatty acids, vitamins, sugars, and proteins, and secondary metabolites produced in trichomes, the cell's factory. Primary metabolites provide nutritional value. Secondary metabolites include approximately 120 terpenoids (61 monoterpenes, 52 sesquiterpenoids, and 5 triterpenoids [2], over 26 flavonoids [2], lignans, stilbenes and derivatives, alkaloids, and 20 steroids [2] dihydrophenanthrenes, glycoproteins, and dibenzyls $[5,6]$. In addition to sharing analgesic, antimicrobial anti-cancer, anti-inflammatory, and neuroprotective properties, a majority also share anti-oxidant capabilities.

During many pathological conditions and diseases processes like age-related inflammatory and autoimmune diseases [7], asthma, atherosclerosis, cancer, chronic obstructive pulmonary disease, hypertension, ischemia/perfusion, diabetes, and HIV dementia [8], the cells of the body accumulate high levels of toxic reactive oxygen species (R.O.S.)/free radicals in comparison to antioxidants [9]. This imbalance can cause significant damage to cell structures [9]. Antioxidants mitigate excessively produced reactive oxygen species within the cells, and in doing so, reduce the oxidative stress in cells [10]. Antioxidants may therefore have therapeutic applicability against many human diseases including but not limited to cancer, viral infections, cardiovascular diseases and inflammatory diseases [10]. Natural antioxidants may also be revered due to their safer side effect profile compared to synthetic antioxidants used in preventative medicine and in the food industry [10][11].

The biosynthesis of the non-cannabinoids in cannabis is driven by pyruvate/acetyl-CoA for the terpenes via the plastidial deoxyxylulose phosphate/methyl-erythritol phosphate (DOXP/MEP) and cytoplasmic MVA pathways [2], and p-coumaril-CoA via the phenylpropanoid pathway [2] for flavonoids [2]. The synthesis of cannabinoids is via the precursor molecule Olivetolic Acid (OLA) and the polyketide pathway, and another precursor molecule, Geranyl Diphosphate (GPP) and the plastidial deoxyxylulose phosphate/methyl-erythritol phosphate (DOXP/MEP) pathway [2].

In addition to having unique organoleptic properties [12], terpenes provide a wide range of therapeutic benefits to humans, secondary metabolites, particularly terpenes and flavonoids, play a primary role in a plant's defenses against hostile environments, herbivores and phytophagous insects. The identified non-cannabinoid metabolites of cannabis are discussed in more detail in the ensuing sections. 


\section{Non-Cannabinoid Compounds}

Cannabinoid and non-cannabinoid phytochemicals possess bioactive and protective properties that are beneficial to human health. In addition to cannabinoids, the Cannabis plant produces hundreds of non-cannabinoid secondary metabolites including approximately 120 terpenoids (61 monoterpenes, 52 sesquiterpenoids, and 5 triterpenoids [2], essential oils, over 26 flavonoids [2] lignans, stilbenoid derivatives, alkaloids, amino acids, spiro-indans, polyphenols, 20 steroids [2], diyhdrophenanthrenes, glycoproteins (like galactose, glucose, mannose and xylose), and dibenzyls [5, 6]. This list also includes a-cannabispiranol, chrysoeriol, 6-prenylapigenin, cannflavin A and b-acetyl cannabispiranol [13].

Flavonoids and terpenoids in particular, have a wide therapeutic window, including but not limited to cancer, inflammation, excessive reactive oxygen species in cells, and enhancement of neuroprotection. In addition to the phytochemicals mentioned below, a 2008 study by Radwan and colleagues identified and isolated six new non-cannabinoid phytochemicals from a high potency Cannabis sativa L. strain. These were 5-acetoxy-6-geranyl3-n-pentyl-1,4-benzoquinone, 4,5-dihydroxy-2,3,6-trimethoxy-9,10-dihydrophenanthrene, 4,7dimethoxy-1,2,5-trihydroxyphenanthrene, cannflavin C, cb-sitosteryl-3-O-b-D-glucopyranoside20-O-palmitate, and 4-hydroxy-2,3,6,7-tetramethoxy-9,10-dihydrophenanthrene [13].

\subsection{Terpenes and their derivatives, terpenoids.}

The word "terpene" was devised in 1866 by August Kekulé, a German organic chemist. Terpenes are hydrocarbons and are made up of isoprene units (5-carbon building blocks)[14], while terpenoids are an oxidized and denatured form of terpenes that differ in that they contain an additional functional group with oxygen [14]. They are not the same, despite being used synonymously. This oxidation occurs during the drying and curing processes when the plant is exposed to open air. Terpenes are typically classified by the number of isoprene units in the molecule. Isoterpene is the only hemiterpenes. Hemiterpenes and hemiterpenoids like prenol and isovaleric acid only have a single isoprene unit. Monoterpenes and monoterpenoids like pinene (most common terpene produced across plant species), limonene, myrcene, geraniol, and terpineol have two isoprene units. Sesquiterpenes and sesquiterpenoids like humulene and farnesol have three isoprene units. Triterpenes, like squalene-the precursors to all steroids [14], have six isoprene units. Sesquiterpenes and tetraterpenes have seven and eight isoprene units, respectively. Polyterpenes and norisoprenoids have multiple isoprene units in their molecule.

As mentioned before, terpenes and terpenoids are synthesized from an isoprenoid precursor Isopentenyl pyrophosphate (IPP). This is done so via the plastidial deoxyxylulose phosphate/methyl-erythritol phosphate (DOXP/MEP) pathway (monoterpenoids), and the Cytoplasmic Mevalonate (MVA) Pathway (sesquiterpenoids, triterpenoids, and sterols) [2]. Along with cannabinoids, terpenes are considered a main physiological marker of secondary metabolites $[15,16]$.

Terpenes are a large and diverse class of aromatic compounds responsible for the unique flavours and scents of many herbs and plants and phenotypic variation across plant species. Over 20,000 terpenes exist across plant species [17], and over 150 alone in the Cannabis plant [18]. This makes Terpenes the largest classification of phytochemicals (Andre, 
Hausman, Guerriero, 2016). Their primary role in plants vary but, in some plants, may attract pollinators, and in others, deter herbivores and inhibit microbial growth. Terpenes and terpenoids are highly concentrated in the essential oils extracted from the Cannabis plant, and are also responsible for the therapeutic benefits that Essential Oils provide.

The most common terpenes in cannabis include limonene, $\alpha$-pinene, $\beta$-pinene, humulene, $\beta$-caryophyllene, linalool and myrcene [2]. Other common terpenes in cannabis include bisabool, borneol, camphene, geraniol, ocimene, terpineol, and valencene. These fall into the monoterpenoids and sesquiterpenoids categories. Examples of mono- and sesquiterpenoids profiled in secondary metabolites in medicinal cannabis inflorescences, leaves, stem bars, and roots include $\alpha$-pinene (monoterpenoid), (-)- $\beta$-pinene (monoterpenoid), camphene (monoterpenoid), sabinene (monoterpenoid), $\beta$-Myrcene (monoterpenoid), $\alpha-$ terpinene (monoterpenoid), linalool (monoterpenoid), eucalyptol (monoterpenoid), limonene (monoterpenoid), $\beta$-Caryophyllene (sesquiterpenoid), $\alpha$-Humulene (sesquiterpenoid), valencene (sesquiterpenoid), $\alpha$-Bisabolol (sesquiterpenoid), and trans-Nerolidol (sesquiterpenoid) [2].

Terpenes provide aromatherapeutic benefits to humans including stress-, anxiety, and depression-relief, decongestion, and a general pharmacologically synergistic effect in combination with cannabinoids and flavonoids. As a result, terpenes are frequently used in cosmeceuticals, perfumes and beverage flavouring. Table 1 below shows the biological properties of other common terpenes found in cannabis.

Table 1 below shows the biological properties of some common terpenes in cannabis.

\begin{tabular}{|c|c|c|}
\hline Terpene & Biological Property & References \\
\hline \multirow[t]{3}{*}{ Myrcene } & Potent analgesic & [19] \\
\hline & $\begin{array}{l}\text { Anti-oxidant, neuroprotective, anti- } \\
\text { inflammatory. }\end{array}$ & [20] \\
\hline & Anticonvulsant & [21] \\
\hline 1,8-cineole & $\begin{array}{l}\text { Increases cerebral blood flow and enhances } \\
\text { cortical activity }\end{array}$ & {$[22]$} \\
\hline \multirow[t]{3}{*}{ Limonene } & $\begin{array}{l}\text { inhibits many species of bacteria and fungi } \\
\text { - repellant. }\end{array}$ & [23] \\
\hline & $\begin{array}{l}\text { Anti-inflammatory, anti-oxidant, anti-viral, } \\
\text { anti-diabetic, anti-cancer }\end{array}$ & {$[20]$} \\
\hline & Anti-depressant; anticonvulsant & {$[21]$} \\
\hline \multirow[t]{3}{*}{$\alpha$-pinene $\&$-pinene } & Antimicrobial; repellant & [23] \\
\hline & $\begin{array}{l}\text { Bronchodilator; } \\
\text { Anti-inflammatory }\end{array}$ & {$[20]$} \\
\hline & $\begin{array}{l}\text { Memory improvement/enhancement; } \\
\text { acetylcholinesterase inhibitor }\end{array}$ & [21] \\
\hline $\begin{array}{l}\text { Linalool } \\
\text { (Lavender scent) }\end{array}$ & $\begin{array}{l}\text { Anxiolytic, anti-inflammatory; anti- } \\
\text { microbial, anti-cancer, neuroprotective, } \\
\text { Antidepressant }\end{array}$ & {$[20]$} \\
\hline
\end{tabular}




\begin{tabular}{|c|c|c|}
\hline & Anti-influenza & [24] \\
\hline & Sedative; Induces apoptosis in cancer cells & {$[21]$} \\
\hline \multirow[t]{7}{*}{$\alpha$-terpineol } & Antimicrobial; repellant & {$[23]$} \\
\hline & Anti-inflammatory & {$[25]$} \\
\hline & Analgesic & {$[26]$} \\
\hline & Nociception-inhibition & {$[26]$} \\
\hline & Anti-convulsant & {$[27]$} \\
\hline & Anti-Microbial & [21] \\
\hline & Gastroprotective & [28] \\
\hline Borneol & Antimicrobial; repellant & {$[23]$} \\
\hline \multirow[t]{2}{*}{$\beta$ - caryophyllene } & $\begin{array}{l}\text { Anti-inflammatory } \\
\text { Analgesic }\end{array}$ & {$[20]$} \\
\hline & Anti-spasmic in gut muscles & [21] \\
\hline Humulene & Anti-allergy; anti-cancer & [20] \\
\hline Ocimene & $\begin{array}{l}\text { Antifungal; Antibacterial; Anti-oxidant; } \\
\text { antiviral; anti-inflammatory }\end{array}$ & {$[29,30,31,32,33]$} \\
\hline
\end{tabular}

The Medical Cannabis Network also reports that a current study is being undertaken by researchers at the Israel Institute of Technology investigating the therapeutic efficacy of a cannabis terpene inhalant formulation in suppressing the immune system response against COVID-19.

\subsection{Phenolic Compounds in Cannabis sativa}

Polyphenols, also known as phenylpropanoids, are a class of over 10,000 chemical compounds that have phenyl groups/rings $\left(\mathrm{C}_{6} \mathrm{H}_{5}\right)$. A phenyl ring consists of a 6-carbon structure formed on a hexagonal plane. Five of these carbon atoms are each individually bonded to hydrogen atoms

A 2020 study by Izzo and colleagues analyzed some phenolic compounds in Cannabis sativa L. inflorescences using UHPLC-Q-Orbitrap HRMS (Ultra-High-Performance Liquid Chromatography-Quadruple-Orbitrap High-Resolution Mass Spectrometry) and report that Cannabisin A, B and C were the predominant phenolic compounds identified [34]. Other phenolic compounds analyzed in the same study include catechin (a flavonol), flavonone (a flavonol), quercetin (a phenolic amide), kaempferol (a phenolic amide), N-transCaffeoyltyramine (a phenolic amide), cannflavin A \& B (flavones), luteolin (a flavone), Apigenin (a flavone), p-Coumaric acid (a phenolic acid), ferulic acid (a phenolic acid), chlorogenic acid (a phenolic acid), and hydroxycinnamic acid (a phenolic acid) [34].

\subsection{Flavonoids}

Flavonoids are a large family of polyphenolic plant compounds that naturally occur in fruits, vegetables, chocolate, and beverages like wine and tea. 
There are six classes of flavonoids including anthocyanidins, flavan-3-ols, flavonols, flavanones, flavones, and isoflavones. Chemically, flavonoids have the general structure of a 15carbon skeleton, which consists of two phenyl rings ( $A$ and $B$ ), and a heterocyclic ring. This carbon structure can be abbreviated C6-C3-C6. Figure 1 below show the structure of a phenyl ring.<smiles>Oc1ccccc1</smiles>

Figure 1. Structure of Phenyl ring. Note 6carbon rings.

Flavonoids are found in a wide range of plants and may act as physiological regulators, cell-cycle inhibitors and/or chemical messengers. They are also responsible for plant pigmentation (flower coloration to attract pollinator animals/insects), UV filtration/protection, and symbiotic nitrogen fixation.

Flavonoids provide color, flavour and aroma. These class of compounds also have anticancer, antioxidant, antithrombogenic, antidiabetic and neuroprotective activities via modulation of a number of cell-signaling cascades.

A 2016 study by Bertoia and colleagues explored the relationship between dietary flavonoid intake (of flavonols, flavones, flavanones, flavan-3-ols, anthocyanins, and flavonoid polymers) and weight change at 4-year intervals between 1986 and 2011 of 124,086 men and women. In conclusion of this study, intake of foods rich in flavonols, flavan-3-ols, anthocyanins, and flavonoid polymers, inversely related to weight gain. Anthocyanidins had the greatest negative correlation with weight maintenance [35]. See tables 2 and 3 below for some biological properties of some common classes of flavonoids.

Table 2 showing biological properties of some common classes of flavonoids.

\begin{tabular}{lll}
\hline \multicolumn{1}{c}{ Flavonoid } & \multicolumn{1}{c}{ Biological Property } & \\
\hline $\begin{array}{l}\text { Flavonols } \\
\text { (E.g. Quercetin \& } \\
\text { Kaempferol) }\end{array}$ & $\begin{array}{l}\text { Antioxidant; } \\
\text { Cardioprotective }\end{array}$ & \\
\hline Flavanones & Antioxidant; Anti-cancer; & [36] \\
& Anti-inflammatory & \\
\hline Isoflavonoids & $\begin{array}{l}\text { Phytoestrogenic (mimic } \\
\text { the hormone estrogen); } \\
\end{array}$ & Hormone balance and \\
& metabolism. \\
\hline
\end{tabular}




\begin{tabular}{lll}
\hline $\begin{array}{l}\text { Anthocyanins } \\
\text { (responsible for a } \\
\text { plant's unique colour) }\end{array}$ & $\begin{array}{l}\text { Antioxidant and Anti- } \\
\text { inflammatory. }\end{array}$ & [36] \\
\hline
\end{tabular}

Over 20 different flavonoids have been identified in Cannabis, most of which fall into flavonol and flavone group [2]. One such flavonoid is Cannflavin A, which is considered to be 30 times more effective than Aspirin at inhibiting prostaglandin E2, a significant modulator of inflammation [37]. Cannflavin B is also an inhibitor of prostaglandin E2 [38].

Another major flavonoid derived from Cannabis is Caflanone. In recent studies, caflanone demonstrated activity against the human coronavirus hCov-OC43 (Covid-19) belonging to clade $b$ of the genus Betacoronavirus same as SARS-COV-2 [38]. In vitro, caflanone inhibited the hCov-OC43 human Coronavirus with an EC50 of $0.42 \mu \mathrm{M}$ [39]. In silico studies showed that caflanone may act by inhibiting the Angiotensin-converting enzyme 2 (ACE2) receptor found in the lung and respiratory tract, and used by the virus during cell entry and infection. Caflanone was also shown to have strong binding affinity to two of the proteases (PLpro \& 3CLpro) are vital to the replication of SARS-COV-2 in humans, thereby inhibiting viral entry to and/or replication within human cell [39].

The docking/binding studies results below show that the phytoantiviral Flavonoids (Hesperetin, Myricetin, Linebacker, and Caflanone) could bind equally or more effectively than CLQ [39].

Table 3. showing more therapeutic benefits of flavonoids.

\begin{tabular}{lc}
\hline \multicolumn{1}{c}{ Therapeutic window/benefits of flavonoids } & Patent Number \\
\hline $\begin{array}{l}\text { Flavonoid derivatives targeting kinases, sirtuins and } \\
\text { oncogenic agents for the treatment of cancers. }\end{array}$ & {$[40,41]$} \\
\hline $\begin{array}{l}\text { Agent containing flavonoid derivatives for treating cancer } \\
\text { and inflammation. }\end{array}$ & {$[42]$} \\
\hline $\begin{array}{l}\text { Therapeutic agents containing cannabis flavonoid } \\
\text { derivative for ocular disorders. }\end{array}$ & {$[43]$} \\
\hline $\begin{array}{l}\text { Therapeutic agents containing cannabis flavonoid } \\
\text { derivatives for the prevention and treatment of } \\
\text { neurodegenerative disorders. }\end{array}$ & {$[44]$} \\
\hline $\begin{array}{l}\text { Pi 4-kinase inhibitor as a therapeutic for viral hepatitis, } \\
\text { cancer, malaria. autoimmune disorders and inflammation, } \\
\text { and a radiosensitizer and immunosuppressant. }\end{array}$ & {$[45]$} \\
\hline $\begin{array}{l}\text { Therapeutic antiviral agents containing cannabis } \\
\text { cannabinoid derivatives. }\end{array}$ & {$[46]$} \\
\hline
\end{tabular}

\subsection{Fatty Acids of Cannabis seeds}

Fatty acids have high nutritional value. They are a class of molecules made up of a chain of carbon atoms bonded with hydrogen atoms, with a function carboxyl group (-COOH) 
attached to a terminal end [47]. It is this functional group that participates in chemical reactions that allow fatty acids to perform their physiological roles. These roles include: providing insulation, storing and providing energy for cells in the absence of glucose, providing the precursor (cholesterol) for the production of hormones and intracellular membranes (estrogen, testosterone, vitamin D hormone, steroids, and prostaglandins), forming the building blocks of glycolipids and phospholipids which form the cell membrane and subcellular membrane, transporting fat soluble vitamins ( $A, D, E$ and $K)$, and modifying proteins.

A 1996 study by Ross and colleagues explored the composition of fatty acids present in the lipid matter of [commercial] cannabis seeds from different countries. Some fatty acids in Commercial Cannabis sativa (seeds) include caproic acid, caprylic acid, myristic acid, palmitoleic acid, palmitic acid, margaric acid, oleic acid, linolenic acid, isolinolenic acid, linoleic acid, stearic acid, eicosenoic aicd, arachidic acid, isoarachidic acid, and behenic acid [48].

It should also be noted that Cannabis sativa seeds, particularly those of the hemp variety, typically have nutritional value. The seed is made up of approximately $25 \%$ high-quality protein and $35 \%$ fat [49]. Hemp oil extracted from the seed contain significant concentrations of fatty acids (particularly omega-6 fatty acids), vitamin C [50][51], thiamine [50][51], riboflavin [50][51], vitamin E [50][51], minerals like calcium [50], magnesium [50][51], potassium [50][51], phosphorus [50][51], iron [50][51]. Zinc [50][51], sodium [50][51], copper [51], and macronutrients likes fat [50], carbs [50], fiber [50], and protein [50].

In comparison to other popular and nutritionally-rich edible oils like canola, sunflower, pumpkin and soybean, only Hemp oil provides the optimal ratio (3:1) of omega-6 fatty acid (Linolic Acid) and omega-3- fatty acids (Alpha-linolenic acid), along with Gamma linolenic acid (GLA) and Stearidonic Acid (SDA) [49].

\subsection{Alkaloids in Cannabis}

In 1876 Preobrajensky claimed to find nicotine in cannabis resin (hashish) from Uzbekistan in 1876 [52]. This was later rejected on the basis that cannabis users from that part of the world tend to mix the resin with tobacco before smoking. This made it likely that the presence of nicotine was due to the tobacco. In later 1881 at the British Pharmaceutical Conference, Siebold and Bradbury reported on the isolation of the alkaloid Cannabinine [53]. Two years later in 1883, Hay isolated Tetanocannabin, another biologically active alkaloid. It was so called because it produced strychnine-like convulsions in frogs [54]. In 1986, even Merck (of Darmstadt) began marketing and advertising a "Cannabine Alkaloid" product [55].

Alkaloids are a class of hetero-cyclical organic compounds that contain one or more nitrogen atom. They may also contain an oxygen, sulfur, chlorine, bromine, or phosphorus atom attached to the molecule. They are most associated with plants, but are also produced by microorganisms and animals [56]. In plants, alkaloids are a form of chemical defense against herbivores. Many alkaloids are pharmacologically active. In fact, alkaloids make up about $60 \%$ of plant-derived drugs [57]. On the same tangent, dndogenous indole alkaloids have been confirmed in hemp [58].

Alkaloids have a variety of therapeutic applications as analgesics, anti-bacterial, anticancer, antiarrythmic, anti-asthma agents, antimalarials, anticholinergics, bronchodilatory, 
laxative, miotic, oxytocic, vasodilatory, psycotropic, and stimulating agents. This class of compounds include morphine, cocaine, nicotine, caffeine, quinine, ephedrine, among others.

Cannabinaceous alkaloids are alkaloids produced by Cannabis sativa. These include, but are not limited to Cannabisativine and Anhydrocannabisativine [59].

A 1971 study by Klein and colleagues investigated the constituents of alkaloids mixtures extracted from Cannabis plants and reported the isolated of four alkaloids, namely Cannabimines A-D [60]. It was also noted that both Cannabimine A and Anyhrocannabisativine (isolated in 1976) share the same molecular formular (C21 H37 N3 O2) [59].

Cannabisativine was the first cannabinaceuous alkaloid to be fully identified. It was isolated in 1975 in Mississippi, from the roots of a Mexican variant of Cannabis sativa [61]. These were isolated from the roots of the Cannabis plant. These alkaloids are antiparasitic, antipyretic, antiemetic, antitumor, diuretic and analgesic [62]. Natural (+)-cannabisativine was totally synthesized from a dihydropyridine derivative [63].

\subsection{Lignanamides and phenolic acids}

Lignanamides are another example of naturally-occurring, non-cannabinoid secondary metabolites with bioactivity. This class of phytochemicals produced in cannabis include Cannabisin A-G, Grossamide [64].

These phytochemicals are powerful anticancer, antitumor, antiviral, antioxidant, antidiabetic, cardiovascular, cytotoxic, antineoplastic, anti-inflammatory, anti-obesity, analgesic, antihyperlipidemic and anti-hyperlipidemic agents [64], and they also inhibit Acetylcholinesterase inhibitory [65]. In this 2015 study by Yan and colleagues, four new lignanamides were also isolated from the hemp seed. These were Cannabisin $\mathrm{M}$, Cannabisin $\mathrm{N}$, Cannabisin O, and 3,3'-demethyl-heliotropamide [65]. Cannabisins are unique to the Cannabis plant. It should also be noted that phenolic amides share these bioactive properties [65].

\subsection{Amino Acids}

Amino acids are the building blocks of proteins which carry out a multiple biological functions in the body including energy production, fat metabolism, muscle metabolism, immune system function, response and maintenance, the synthesis of enzymes, hormones and neurotransmitters, tissue growth, nutrient absorption, sleep-wake cycles, regulation of blood sugar levels, hemoglobin production, digestion, and sexual function [66]. The amino acid molecule consists of a central carbon atom, an amino group (-NH2), a function $\mathrm{R}$ group side chain that defines the chemical properties of that specific amino acid, and a carboxyl group (COOH) [67].

Cannabis sativa (hemp) seeds contain eight of the nine essential amino acids. These include histidine, isoleucine, leucine, lysine, methionine, phenylalanine, threonine, and valine. Tryptophan, from which serotonin (the "happy" neurotransmitter), is not produced in the Cannabis sativa plant. In fact, a 2007 study by Bambico and colleagues suggests that cannabinoids (THC, CBD) may exhibit potent antidepressant properties at low doses, and depressant properties at high doses [68]. This may explain the depressant properties of Cannabis at certain doses. Essential amino acids are defined as those that cannot be 
synthesized in our bodies, but instead have to be obtained through food. A 2014 study by Audu, Ofojekwu, Ujah \& Ajima suggest that amino acids might be most concentrated within the leaves of the Cannabis sativa plant [69]. Some amino acids found in the leaf, stem and seeds of Cannabis sativa include lysine, histidine, arginine, aspartic acid, threonine, serine, glutamic acid, proline, glycine, alanine, cystine, valine, methionine, isoleucine, leucine, norleucine, tyrosine, and phenylalanine [69].

Hempseed protein is unique within the plant kingdom because it contains the highest composition (65\%) of the globulin edistin which make up many enzymes, antibodies, and hormones in the body [70].

\subsection{Stilbenes and stilbenoids}

Stilbenes (and their derivatives) are naturally occurring polyphenolic phytochemicals that, aside from being a form of herbivore- and disease-resistance mechanisms for many lower and higher plants in the plant kingdom [71], have a wide range of biological activity and medicinal value to humans [72]. Stilbenoids are stilbene derivates that have been hydroxylated [73].

A 2004 study by El-Feraly isolated, characterized and synthesized dihydroresveratrol (3,5,4'-Trihydroxybibenzyl), a metabolite derivative of resveratrol, a dihydrostilbenoid, from Cannabis sativa [74]. A 2019 study by Giménez-Bastida and colleagues reported that dihydroresveratrol and other metabolite derivatives of resveratrol, induced senescence in breast cancer cells [75].

Nineteen stilbenoids unique to the cannabis plant were identified in a 1995 study by Ross and ElSohly [5]. These can be divided into three main structural classes: eleven spiroindans, eight dihydrostilbenes/bibenzyls, five phenanthrenes and prenylated, geranylated and glycosydated derivates [3][64]. Some spiroindans include Cannabispirone, cannabispirenone- $B, \beta$-cannabispiranol, $\alpha$-cannabispiranol [64]. Some dihydrostilbenes include canniprene [prenylated], cannabistilbene I [prenylated], cannabistilbene Ila [prenylated], cannabistilbene IIb [prenylated], dihydroresveratrol [non-prenylated], 3,3'-dihydroxy-5,4'dimethoxy bibenzyl [non-prenylated], and 3,4'-dihydroxy-5-methoxy bibenzyl [non-prenylated] [64].

Phenanthrenes like 9,10-dihydrophenanthrene, 4,7-dimethoxy-1,2,5trihydroxyphenanthrene, 4,5-dihydroxy-2,3,6-trimethoxy-9,10-dihydrophenanthrene, 4hydroxy-2,3,6,7-tetramethoxy-9,10-dihydrophenanthrene, and Denbinobin (5-hydroxy-3,7dimethoxy-1,4-phenanthrenequinone) have also been isolated from Cannabis sativa, but these are not unique to the plant [64].

Denbinoben, first isolated in a 2008 study by Sànchez-Duffhues, is reported to have beneficial bioactive properties to human health as an anti-HIV, antioxidant, anti-tumor, and inhibitor of platelet aggregation [76].

\section{Conclusion and future prospects}

The Cannabis plant produces both cannabinoid and non-cannabinoid phytochemicals of great economic and medicinal value [1]. These include some 104 cannabinoids (including CBD, 
$\Delta^{9}$-THC, and CBG), 120 terpenoids, over 26 flavonoids [1], lignans, phenolic amides, amino acids, nitrogenous compounds, steroids, fatty acids, alkaloids, stilbenes, vitamins, minerals and other phytochemicals. These phytochemicals are produced in virtually all areas of the plant (root, leaves, stem). The aforementioned Cannabinoids and non-cannabinoid secondary metabolites are produced primarily in the trichomes of the Cannabis plant [77].

Cannabinoid and non-cannabinoid secondary metabolites have wide therapeutic application across many ailments including different types of cancers, diabetes, cardiovascular disorders, neurodegenerative disorders, inflammation-related diseases, and viral infections. Unlike THC, one of the most studied cannabinoids, the majority of these phytochemicals are non-psychotropic. This means they will not produce the psychoactive effects produced by THC, but will still have therapeutic benefit.

A majority of these phytochemicals require further in-depth characterization for their therapeutic efficacy and safety. There is also the need for a more rigorous standardization of cannabis cultivation practices so as to ensure consistent reproducibility of the profiles of secondary metabolites like terpenes. The ability to reconstruct synthetic pathways, elucidate regulatory mechanism involved in the production of secondary metabolites, and establishing genomic, metabolomic, transcriptomic maps/"fingerprints" of said secondary metabolites will allow drug-manufacturers to produce more targeted drugs [78].

There is a need for more systematic botanical, physicochemical and chemical analyses of the Cannabis plant. Further research is required to determine the efficacy, dosage standards, optimum extraction methods/solvents, cytotoxicity/hepatoxicity, pharmacokinetics, molecular mechanisms of action, phytoantiviral screening methods, and drug interactions for many phytochemicals in the Cannabis plant.

Author Contributions: Conceptualization, H.L., and B.S., Writing, review and editing: H.L., B.S., J.B., N.T., and W.N.

Funding: This research received no external funding.

Conflicts of Interest: Authors H.L. and N.T. are employees of Vilotos and Flavocure and both companies have commercial interest in Caflanone. The other authors declare no conflicts of interest. 


\section{References}

1. Li, H. (1973). An archaeological and historical account of cannabis in China. Economic Botany, 28(4), 437-448. doi:10.1007/bf02862859

2. Jin, D., Dai, K., Xie, Z., \& Chen, J. (2020). Secondary Metabolites Profiled in Cannabis Inflorescences, Leaves, Stem Barks, and Roots for Medicinal Purposes. Scientific Reports, 10(1). doi:10.1038/s41598-020-60172-6

3. Pollastro, F., Minassi, A., Fresu, L. (2018). Cannabis Phenolics and their Bioactivities. Current medicinal chemistry. 24. 10.2174/0929867324666170810164636.

4. Kaur, R., Matta, T., \& Kaur, H. (2017). Plant Derived Alkaloids. Haya: The Saudi Journal of Life Sciences, 2(5), 158-189

5. Ross SA, ElSohly MA (1995) Constituents of Cannabis sativa L. XXVIII a review of the natural constituents: 1980-1994. Zagazig J Pharm Sci 4:1-10

6. Turner CE, ElSohly MA, Boeren EG (1980) Constituents of Cannabis sativa L. XVII: a review of the natural constituents. J Nat Prod 43:169-243

7. Grimaldi, C., Caputo, M., Tecce, M., \& Capasso, A. (2013). 13. Cannabinoids as antioxidant: An overview.

8. Hampson, A. J., Grimaldi, M., Lolic, M., Wink, D., Rosenthal, R., \& Axelrod, J. (2000). Neuroprotective antioxidants from marijuana. Annals of the New York Academy of Sciences, 899, 274-282.

9. Birben, E., Sahiner, U. M., Sackesen, C., Erzurum, S., \& Kalayci, O. (2012). Oxidative stress and antioxidant defense. The World Allergy Organization journal, 5(1), 9-19. https://doi.org/10.1097/WOX.0b013e3182439613

10. Krishnaiah, D., Sarbatly, R., \& Nithyanandam, R. (2011). A review of the antioxidant potential of medicinal plant species. Food and Bioproducts Processing, 89(3), 217-233. doi:10.1016/j.fbp.2010.04.008

11. Cañadas, Raquel \& Gonzalez-Miquel, Maria \& González, Emilio \& Díaz, Ismael \& Rodriguez, Manuel. (2020). Overview of neoteric solvents as extractants in food industry: A focus on phenolic compounds separation from liquid streams. Food Research International. 136. 109558. 10.1016/j.foodres.2020.109558.

12. Booth, J. K., Page, J. E., \& Bohlmann, J. (2017). Terpene synthases from Cannabis sativa. Plos One, 12(3). doi:10.1371/journal.pone.0173911

13. Radwan, M. M., Elsohly, M. A., Slade, D., Ahmed, S. A., Wilson, L., El-Alfy, A. T., Khan, I. A., \& Ross, S. A. (2008). Non-cannabinoid constituents from a high potency Cannabis sativa variety. Phytochemistry, 69(14), 2627-2633. https://doi.org/10.1016/j.phytochem.2008.07.010

14. Al-Taweel, A. (2018). Chapter 1. In 962750137748733601 S. Perveen (Author), Terpenes and terpenoids. London: IntechOpen.

15. Fischedick,J.T.,Hazekamp,A.,Erkelens, T.,Choi,Y.H.,and Verpoorte,R.(2010). Metabolic fingerprinting of Cannabis sativa L., cannabinoids and terpenoids for chemotaxonomic and drug standardization purposes. Phytochemistry 71, 20582073.doi:10.1016/j.phytochem.2010.10.001 
16. Elzinga,S.,Fischedick,J.,Podkolinski,R.,andRaber,J.C.(2015).Cannabinoids and terpenes as chemotaxonomic markers in cannabis. Nat.Prod.Chem.Res. 3:81. doi:10.4172/23296836.1000181

17. Jacobs, M. (2020, July 27). The Difference Between Cannabinoids and Terpenes (Analytical Cannabis). Retrieved July 28, 2020, from https://www.analyticalcannabis.com/articles/the-difference-between-cannabinoidsand-terpenes-311502

18. Booth, J. K., \& Bohlmann, J. (2019). Terpenes in Cannabis sativa - From plant genome to humans. Plant Science, 284, 67-72. doi:10.1016/j.plantsci.2019.03.022

19. Rao, V. S., Menezes, A. M., \& Viana, G. S. (1990). Effect of myrcene on nociception in mice. Journal of Pharmacy and Pharmacology, 42(12), 877-878. doi:10.1111/j.20427158.1990.tb07046.x

20. Johnson, J., \& Theisen, E. (2020, March 6). What are terpenes? Retrieved July 28, 2020, from https://www.medicalnewstoday.com/articles/what-are-terpenes

21. McPartland, J. M., \& Russo, E. B. (2014). Non-Phytocannabinoid Constituents of Cannabis and Herbal Synergy. Handbook of Cannabis, 280-295. doi:10.1093/acprof:oso/9780199662685.003.0015

22. Ambrosch, S., Duliban, C., Heger, H., Moser, E., Laistler, E., Windischberger, C., \& Heuberger, E. (2018). Effects of 1,8-Cineole and (-)-Linalool on Functional Brain Activation in a Working Memory Task. Flavour and Fragrance Journal, 33(3), 235-244. doi:10.1002/ffj.3436

23. Vieira-Brock, P. L., Vaughan, B. M., \& Vollmer, D. L. (2017). Comparison of antimicrobial activities of natural essential oils and synthetic fragrances against selected environmental pathogens. Biochimie Open, 5, 8-13. doi:10.1016/j.biopen.2017.09.001

24. Choi, H. (2018). Chemical Constituents of Essential Oils Possessing Anti-Influenza A/WS/33 Virus Activity. Osong Public Health and Research Perspectives, 9(6), 348-353. doi:10.24171/j.phrp.2018.9.6.09

25. Held, S., Schieberle, P., \& Somoza, V. (2007). Characterization of $\alpha$-Terpineol as an Antiinflammatory Component of Orange Juice by in Vitro Studies Using Oral Buccal Cells. Journal of Agricultural and Food Chemistry, 55(20), 8040-8046. doi:10.1021/jf071691m

26. Quintans-Júnior, L. J., Oliveira, M. G., Santana, M. F., Santana, M. T., Guimarães, A. G., Siqueira, J. S., .. . Almeida, R. N. (2011). $\alpha$-Terpineol reduces nociceptive behavior in mice. Pharmaceutical Biology, 49(6), 583-586. doi:10.3109/13880209.2010.529616

27. Sousa, D. P., Quintans, L., \& Almeida, R. N. (2007). Evolution of the Anticonvulsant Activity of $\alpha$-Terpineol. Pharmaceutical Biology, 45(1), 69-70. doi:10.1080/13880200601028388

28. Souza, R., Cardoso, M., Menezes, C., Silva, J., De Sousa, D., \& Batista, J. (2011). Gastroprotective activity of $\alpha$-terpineol in two experimental models of gastric ulcer in rats. DARU : Journal of Faculty of Pharmacy, Tehran University of Medical Sciences, 19(4), 277-281.

29. Valente, J., Zuzarte, M., Gonçalves, M. J., Lopes, M. C., Cavaleiro, C., Salgueiro, L., \& Cruz, M. T. (2013). Antifungal, antioxidant and anti-inflammatory activities of Oenanthe crocata L. essential oil. Food and chemical toxicology : an international journal published 
for the British Industrial Biological Research Association, 62, 349-354. https://doi.org/10.1016/j.fct.2013.08.083

30. Cavaleiro, C., Salgueiro, L., Gonçalves, M. J., Hrimpeng, K., Pinto, J., \& Pinto, E. (2015). Antifungal activity of the essential oil of Angelica major against Candida, Cryptococcus, Aspergillus and dermatophyte species. Journal of natural medicines, 69(2), 241-248. https://doi.org/10.1007/s11418-014-0884-2

31. Golfakhrabadi, F., Khanavi, M., Ostad, S. N., Saeidnia, S., Vatandoost, H., Abai, M. R., Hafizi, M., Yousefbeyk, F., Rad, Y. R., Baghenegadian, A., \& Ardekani, M. R. (2014). Biological Activities and Composition of Ferulago carduchorum Essential Oil. Journal of arthropod-borne diseases, 9(1), 104-115.

32. Kim, M. J., Yang, K. W., Kim, S. S., Park, S. M., Park, K. J., Kim, K. S., Choi, Y. H., Cho, K. K., \& Hyun, C. G. (2014). Chemical composition and anti-inflammation activity of essential oils from Citrus unshiu flower. Natural product communications, 9(5), 727-730.

33. Loizzo, M. R., Saab, A. M., Tundis, R., Statti, G. A., Menichini, F., Lampronti, I., Gambari, R., Cinatl, J., \& Doerr, H. W. (2008). Phytochemical analysis and in vitro antiviral activities of the essential oils of seven Lebanon species. Chemistry \& biodiversity, 5(3), 461-470. https://doi.org/10.1002/cbdv.200890045

34. Izzo, L., Castaldo, L., Narváez, A., Graziani, G., Gaspari, A., Rodríguez-Carrasco, Y., \& Ritieni, A. (2020). Analysis of Phenolic Compounds in Commercial Cannabis sativa L. Inflorescences Using UHPLC-Q-Orbitrap HRMS. Molecules, 25(3), 631. doi:10.3390/molecules25030631

35. Bertoia, Monica \& Rimm, Eric \& Mukamal, Kenneth \& Hu, Frank \& Willett, Walter \& Cassidy, Aedín. (2016). Dietary flavonoid intake and weight maintenance: Three prospective cohorts of 124,086 US men and women followed for up to 24 years. BMJ. 352. i17. 10.1136/bmj.i17.

36. Richter, N. (2020, March 10). What are Flavonoids? [Comprehensive Guide]. Retrieved July 28, 2020, from https://wayofleaf.com/education/what-are-flavonoids

37. Barrett, M.L.; Gordon, D.; Evans, F.J. (1985). "Isolation from cannabis sativa L. Of cannflavin-a novel inhibitor of prostaglandin production". Biochemical Pharmacology. 34 (11): 2019-2024. doi:10.1016/0006-2952(85)90325-9. PMID 3859295.

38. Werz, O., Seegers, J., Schaible, A. M., Weinigel, C., Barz, D., Koeberle, A., . . Appendino, G. (2014). Cannflavins from hemp sprouts, a novel cannabinoid-free hemp food product, target microsomal prostaglandin E2 synthase-1 and 5-lipoxygenase. PharmaNutrition, 2(3), 53-60. doi:10.1016/j.phanu.2014.05.001

39. Ngwa, W., Kumar, R., Thompson, D., Lyerly, W., Moore, R., Reid, T., . . . Toyang, N. (2020). Potential of Flavonoid-Inspired Phytomedicines against COVID19. Molecules, 25(11), 2707. doi:10.3390/molecules 25112707

40. U.S. Patent No. US20180098961A1. (n.d.). Washington, DC: U.S. Patent and Trademark Office.

41. U.S. Patent No. WO2017091837A2. (n.d.). Washington, DC: U.S. Patent and Trademark Office.

42. U.S. Patent No. US20170360744A1. (n.d.). Washington, DC: U.S. Patent and Trademark Office. 
43. U.S. Patent No. US10278950B2. (n.d.). Washington, DC: U.S. Patent and Trademark Office.

44. U.S. Patent No. US20190083452A1. (n.d.). Washington, DC: U.S. Patent and Trademark Office.

45. U.S. Patent No. US20190358196A1. (n.d.). Washington, DC: U.S. Patent and Trademark Office.

46. U.S. Patent No. US20180214389A1. (n.d.). Washington, DC: U.S. Patent and Trademark Office.

47. Pertwee, R. G. (2014). Handbook of cannabis: Edited by Roger G. Pertwee. New York: Oxford University Press.

48. Ross, S.A., ElSohly, H.N., ElKashoury, E.A. and ElSohly, M.A. (1996), Fatty Acids of Cannabis Seeds. Phytochem. Anal., 7: 279-283. doi:10.1002/(SICI)10991565(199611)7:6<279::AID-PCA322>3.0.CO;2-P

49. Hazekamp, A., Fischedick, J., Llano-Diez, M., Lubbe, A., Ruhaak, Lucia. (2010). Chemistry of Cannabis. 10.1016/B978-008045382-8.00091-5.

50. Cooke, J. (2016, March 12). Does Cannabis Have Nutritional Benefits? Retrieved July 28, 2020, from https://thesunlightexperiment.com/blog/cannabis-nutritional-benefits

51. Pontassuglia, L., \& Dongo, D. (2019, March 19). Hemp, the Italian superfood - Hemp. Retrieved July 28, 2020, from https://www.greatitalianfoodtrade.it/en/markets/hempthe-italian-superfood

52. Preobrajensky, B., 1876. The alkaloids of Indian hemp and their preparations. Thesis, St Petersburg.

53. L. Siebold and T. Bradbury, Pharm. J . 41, 326 (1881).

54. Hay, M., 1883. A new alkaloid in Cannabis indica. Pharm. J. Trans. 13, 996-997.

55. Merck Index, 1896.

56. Roberts, M. F. (Margaret F.) (1998). Alkaloids : Biochemistry, Ecology, and Medicinal Applications. Wink, Michael. Boston, MA: Springer US. ISBN 9781475729054. OCLC 851770197.

57. Gowda, L., \& Venkategowda, A. (2014). Isolation \& characterization of antimicrobial alklaoids from Plumeria albaflowers against food borne pathogens. American Journal of Life Sciences, 2(6-1),1-6.

58. Yan, X., Zhou, Y., Tang, J., Ji, M., Lou, H., \& Fan, P. (2016). Diketopiperazine indole alkaloids from hemp seed. Phytochemistry Letters, 18, 77-82.

doi:10.1016/j.phytol.2016.09.001

59. Mechoulam, R., \& Hanuš, L. (2000). A historical overview of chemical research on cannabinoids. Chemistry and Physics of Lipids, 108(1-2), 1-13. doi:10.1016/s00093084(00)00184-5

60. Klein, F.K., Rapoport, H., Elliott, H.W., 1971. Cannabis alkaloids. Nature 232, 258-259.

61. Lotter, H.L., Abraham, D.J., Turner, C.E., Knapp, J.E., Schiff, P.L., Jr, Slatkin, D.J., 1975. Cannabisativine. A new alkaloid from Cannabis sati6a L. root. Tetrahedron Lett. 33, 2815-2818.

62. Wink, M. (2020). Potential of DNA Intercalating Alkaloids and Other Plant Secondary Metabolites against SARS-CoV-2 Causing COVID-19. Diversity, 12(5), 175. doi:10.3390/d12050175 
63. Kuethe, J. T., \& Comins, D. L. (2004). Asymmetric Total Synthesis of (+)-Cannabisativine (I). ChemInform, 35(49). doi: 10.1021/jo049724

64. Flores-Sánchez, Isvett Josefina and Robert Verpoorte. "Secondary metabolism in cannabis." Phytochemistry Reviews 7 (2008): 615-639.

65. Yan, X., Tang, J., Passos, C., Nurisso, A., Simões-Pires, C., Ji, M., Lou, H., \& Fan, P. (2015). Characterization of Lignanamides from Hemp (Cannabis sativa L.) Seed and Their Antioxidant and Acetylcholinesterase Inhibitory Activities. Journal of Agricultural and Food Chemistry. 63. 10.1021/acs.jafc.5b05282.

66. Kubala, J. (2018, June 12). Essential Amino Acids: Definition, Benefits and Food Sources. Retrieved July 28, 2020, from https://www.healthline.com/nutrition/essential-aminoacids

67. Abbass, Jad. (2018). Secondary Structure-Based Template Selection for FragmentAssembly Protein Structure Prediction.

68. Bambico, F. R., Katz, N., Debonnel, G., \& Gobbi, G. (2007). Cannabinoids Elicit Antidepressant-Like Behavior and Activate Serotonergic Neurons through the Medial Prefrontal Cortex. Journal of Neuroscience, 27(43), 11700-11711. doi:10.1523/jneurosci.1636-07.2007

69. Audu, B.S., Ofojekwu, P.C., Ujah, A., Ajima, M.N.O. (2014): Phytochemical, proximate composition, amino acid profile and characterization of Marijuana (Cannabis sativa L.) The Journal of Phytopharmacology 3(1): 35-43. - Scientific Figure on ResearchGate. Available from: https://www.researchgate.net/figure/Amino-acid-composition-of-theleaf-stem-and-seeds-of-Cannabis-sativa-obtained-from_tbl3_273692476 [accessed 26 Jul, 2020]

70. Osburn, L. (1992). HEMP SEED: THE MOST NUTRITIONALLY COMPLETE FOOD SOURCE IN THE WORLD. Hemp Line Journal, 1(1), 14-15.

71. Orsini F., Verotta L. (1999) Stilbenes and Bibenzyls with Potential Anticancer or Chemopreventive Activity. In: Zappia V., Della Ragione F., Barbarisi A., Russo G.L., lacovo R.D. (eds) Advances in Nutrition and Cancer 2. Advances in Experimental Medicine and Biology, vol 472. Springer, Boston, MA. https://doi.org/10.1007/978-1-4757-3230-6_15

72. Baur, J., Sinclair, D. Therapeutic potential of resveratrol: the in vivo evidence. Nat Rev Drug Discov 5, 493-506 (2006). https://doi.org/10.1038/nrd2060

73. Montané, X., Kowalczyk, O., Reig-Vano, B., Bajek, A., Roszkowski, K., Tomczyk, R., . . . Tylkowski, B. (2020). Current Perspectives of the Applications of Polyphenols and Flavonoids in Cancer Therapy. Molecules, 25(15), 3342. doi:10.3390/molecules25153342

74. El-Feraly, Farouk. (2004). Isolation, Characterization, and Synthesis of 3,5,4'Trihydroxybibenzyl from Cannabis sativa. Journal of Natural Products - J NAT PROD. 47. 10.1021/np50031a011.

75. Giménez-Bastida JA, Ávila-Gálvez MÁ, Espín JC, González-Sarrías A. Conjugated Physiological Resveratrol Metabolites Induce Senescence in Breast Cancer Cells: Role of p53/p21 and p16/Rb Pathways, and ABC Transporters. Mol Nutr Food Res. 2019;63(22):e1900629. doi:10.1002/mnfr.201900629

76. Sànchez-Duffhues, G.; Calzado, M. A.; de Vinuesa, A. G.; Caballero, F. J.; Ech-Chahad, A.; Appendino, G.; Krohn, K.; Fiebich, B. L.; Muñoz, E. Denbinobin, a naturally occurring 1,4- 
phenanthrenequinone, inhibits HIV-1 replication through an NF-kB-dependent pathway. Biochem. Pharmacol.2008, 76, $1240-1250$.

77. Gonçalves, J., Rosado, T., Soares, S., Simão, A., Caramelo, D., Luís, Â, . . Duarte, A. (2019). Cannabis and Its Secondary Metabolites: Their Use as Therapeutic Drugs, Toxicological Aspects, and Analytical Determination. Medicines, 6(1), 31. doi:10.3390/medicines6010031

78. Andre, C., Hausman, J.F., Guerriero, G. (2016). Cannabis sativa: The Plant of the Thousand and One Molecules. Frontiers in Plant Science. 7. 10.3389/fpls.2016.00019. 